\title{
Hole Scattering in GaSb: Scattering on Space Charge Regions Versus Dipole Scattering
}

\author{
B. PŐDÖR* \\ Budapest Tech, Kandó Kálmán Faculty of Electrical Engineering \\ Institute of Microelectronics and Technology \\ Taraszmező u. 17, 1084 Budapest, Hungary \\ and \\ Hungarian Academy of Sciences \\ Research Institute for Technical Physics and Materials Science \\ P.O.B. 49, 1525 Budapest, Hungary
}

Hole concentration and mobility were investigated by Hall measurements in nominally undoped $p$-type GaSb in the temperature range from 77 to $300 \mathrm{~K}$. The dependence of the thermal ionization energy of native acceptors on the acceptor centre concentration and on the compensation degree was determined. The temperature dependence of the hole mobility was analyzed using a heuristic semi-empirical model as well as using a phenomenological two-hole band model. Space charge scattering and/or dipole scattering described with a mobility contribution with a $\sim T^{-1 / 2}$ like temperature dependence dominated the hole mobility in the investigated temperature range.

PACS numbers: 72.20.Fr, 72.20.Dp, 72.80.Ey

\section{Introduction}

GaSb and related alloys and heterostructures are promising materials for optoelectronic semiconductor devices in the near infrared. Notwithstanding their great potential, GaSb materials are not yet as developed as other III-V compounds like GaAs or InP. Much effort is still needed to elucidate the effects of various defects and impurities on the electrical properties and in a wider sense,

*e-mail: podor.balint@kvk.bmf.hu 
on the general physical properties of GaSb. Undoped GaSb is usually $p$-type $\left(p \approx 10^{16}-10^{17} \mathrm{~cm}^{-3}\right.$ ) with residual acceptor that is related to Ga excess. This acceptor has been associated to the $\mathrm{V}_{\mathrm{Ga}} \mathrm{GaSb}$ complex [1] with optical ionization energy of $\sim 34 \mathrm{meV}[2,3]$.

In this work an experimental and theoretical investigation of the temperature dependence of hole mobility is presented in bulk GaSb, whose electrical properties are determined by native acceptors.

\section{Measurements and results}

Hall data were measured in the Van der Pauw configuration between 300 and $77 \mathrm{~K}$ on 18 samples of undoped $p$-type bulk grown GaSb obtained from three different sources $[4,5]$. The magnetic field was $\sim 0.45 \mathrm{~T}$. The room temperature hole concentration of the samples studied was in the range from $6 \times 10^{16} \mathrm{~cm}^{-3}$ to $1 \times 10^{18} \mathrm{~cm}^{-3}$, the corresponding Hall mobility was between $420 \mathrm{~cm}^{2} /(\mathrm{V} \mathrm{s})$ and $660 \mathrm{~cm}^{2} /(\mathrm{V} \mathrm{s})$ at $300 \mathrm{~K}$ and between $400 \mathrm{~cm}^{2} /(\mathrm{V} \mathrm{s})$ and $2300 \mathrm{~cm}^{2} /(\mathrm{V} \mathrm{s})$ at $77 \mathrm{~K}$.

Representative data for hole concentration $p=1 / e R_{\mathrm{H}}$ in a function of the reciprocal temperature are shown in Fig. 1, while Fig. 2 presents the corresponding

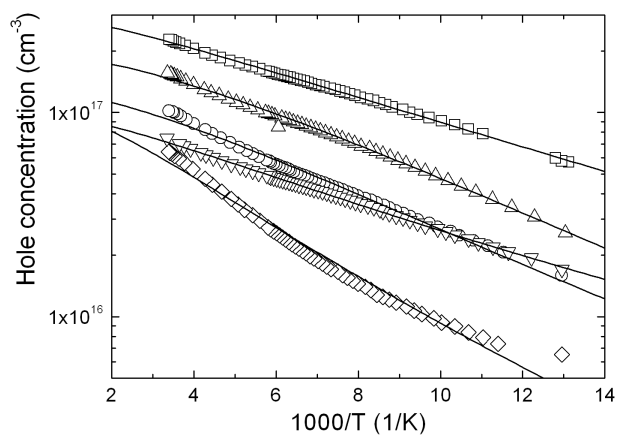

Fig. 1. Hole concentration $p=1 / e R_{\mathrm{H}}$ versus reciprocal temperature. Full lines are theoretical fits with parameters listed in Table I.

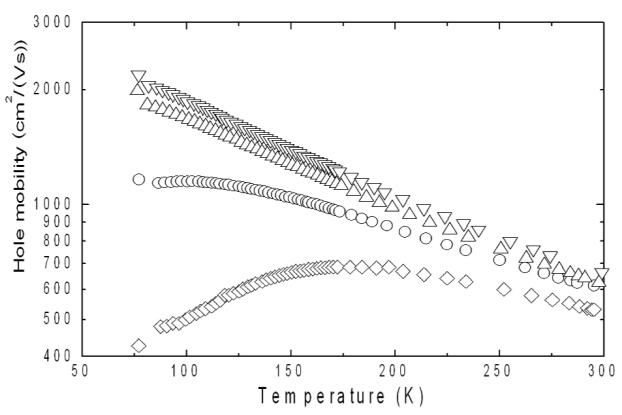

Fig. 2. Temperature variation of the hole mobility. Notations as in Fig. 1. 
TABLE I

Electrical parameters of GaSb single crystals.

\begin{tabular}{c|c|c|c|c|c|c|c}
\hline \hline Sample & $\begin{array}{c}p(300 \mathrm{~K}) \\
{\left[\mathrm{cm}^{-3}\right]}\end{array}$ & $\begin{array}{c}\mu(300 \mathrm{~K}) \\
{\left[\mathrm{cm}^{2} /(\mathrm{V} \mathrm{s})\right]}\end{array}$ & $\begin{array}{c}N_{\mathrm{A}} \\
{\left[\mathrm{cm}^{-3}\right]}\end{array}$ & $\begin{array}{c}N_{\mathrm{D}} \\
{\left[\mathrm{cm}^{-3}\right]}\end{array}$ & $K$ & $\begin{array}{c}E_{\mathrm{A}} \\
{[\mathrm{meV}]}\end{array}$ & $\begin{array}{c}Q N_{\mathrm{SC}} \\
{\left[\mathrm{cm}^{-1}\right]}\end{array}$ \\
\hline $\mathrm{A}$ & $2.2 \times 10^{17}$ & 560 & $7.0 \times 10^{17}$ & $3.9 \times 10^{17}$ & 0.56 & 6.4 & $1.6 \times 10^{5}$ \\
$\mathrm{~B}$ & $1.6 \times 10^{17}$ & 620 & $5.1 \times 10^{17}$ & $3.2 \times 10^{17}$ & 0.62 & 11.1 & $1.5 \times 10^{5}$ \\
$\mathrm{C}$ & $1.0 \times 10^{17}$ & 610 & $6.6 \times 10^{17}$ & $5.3 \times 10^{17}$ & 0.80 & 9.4 & $1.4 \times 10^{5}$ \\
$\mathrm{D}$ & $7.5 \times 10^{16}$ & 660 & $8.0 \times 10^{17}$ & $7 \times 10^{17}$ & 0.87 & 4.4 & $1.4 \times 10^{5}$ \\
$\mathrm{E}$ & $6.4 \times 10^{16}$ & 530 & $13.5 \times 10^{17}$ & $12.4 \times 10^{15}$ & 0.92 & 11.3 & $<0.3 \times 10^{5}$
\end{tabular}

data for the mobility in a function of temperature. Relevant parameters of the samples are collected in Table I.

\section{Hall analysis}

The Hall data were analyzed using a single-acceptor/single-donor model (which gave a satisfactory fit), with a density-of-states effective mass of $m^{*} / m_{0}=0.5$ and an acceptor degeneracy factor of 4 . Where necessary, allowance was made for the partial Fermi degeneracy using standard approximations [6]. The resulting parameters were in the range $N_{\mathrm{A}}=4 \times 10^{17}-1.3 \times 10^{18} \mathrm{~cm}^{-3}, K=$ $0.55-0.90$, and $E_{\mathrm{A}}=3-12 \mathrm{meV}$ (c.f. Table I). The typical errors in the fitted values of $N_{\mathrm{A}}, N_{\mathrm{D}}$, and $E_{\mathrm{A}}$ were 5-15 percent, 10-20 percent, and $0.5-1 \mathrm{meV}$, respectively.

In agreement with the literature the thermal ionization energy $E_{\mathrm{A}}$ of the native acceptor centres deduced from the analysis of temperature dependent Hall data is substantially smaller than their optical ionization energy. The dependence of $E_{\mathrm{A}}$ on the acceptor concentration $N_{\mathrm{A}}$ and on the compensation degree $K$ can be modelled using the general relationship [7-9]

$$
E_{\mathrm{A}}=E_{\mathrm{A}}\left(N_{\mathrm{A}}\right)=E_{\mathrm{A} 0}-f(K) \frac{e^{2}}{4 \pi \varepsilon_{\mathrm{r}} \varepsilon_{0}} N_{\mathrm{A}}^{1 / 3},
$$

here $E_{\mathrm{A} 0}$ is the ionization energy of acceptors at infinite dilution, $N_{\mathrm{A}}$ is the acceptor concentration, $K$ is the compensation degree $\left(K=N_{\mathrm{D}} / N_{\mathrm{A}}, \varepsilon_{0}\right.$ is the dielectric permittivity of the vacuum, and $\varepsilon_{\mathrm{r}}$ is the static dielectric constant of the host semiconductor. $f(K)$ is a (dimensionless) function, whose exact form depends on the details of the concrete mechanism leading to the reduction of the thermal ionization energy.

In Fig. 3 the values of $f(K)=\left(E_{\mathrm{A} 0}-E_{\mathrm{A}}\right)\left(4 \pi \varepsilon_{\mathrm{r}} \varepsilon_{0} / e^{2}\right) / N_{\mathrm{A}}^{1 / 3}$ deduced from the experiments are plotted in a function of the compensation degree $K$ with $E_{\mathrm{A} 0}=34.5 \mathrm{meV}$ and $\varepsilon_{\mathrm{r}}=15.7[1-3]$ alongside with the relevant theoretical relationships $[10,12]$. Besides our results, some data culled from [13-16] are also included. The data displayed exhibit the $f(K) \sim K^{1 / 3}$ like dependence, as implied by the models based on electrostatic interaction [10-12]. An envelope fit to 


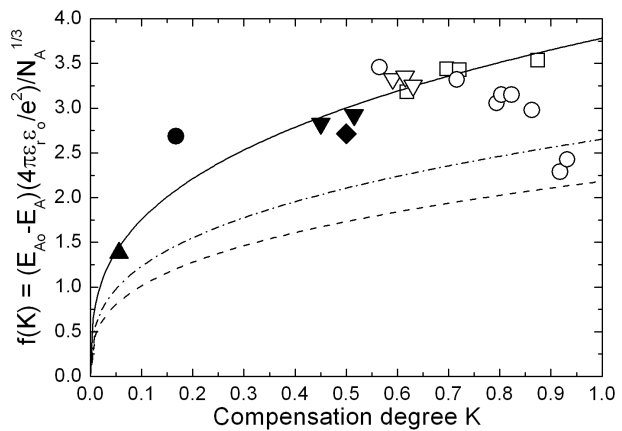

Fig. 3. Theoretical and "experimental" curves of the function $f(K)$ versus $K$. Open squares and circles - this work, open triangles — data from [5], up full triangle data of [13], full circle — data of [14], diamond — data of [15], down full triangles data of [16]. Theoretical curves: full line - fit of electrostatic interaction model to our data, dash-dot line - model of [11], dash line - model of [12].

our data gave a pre-factor of $3.78 \pm 0.05$. This is about 1.4 and 1.7 times larger respectively than the results of two model predictions $f(K)=2.6533 K^{1 / 3}$ [11] and $f(K)=2.1828 K^{1 / 3}[12]$, respectively. Our results therefore indicate a stronger decrease in the thermal ionization energy with increasing acceptor concentration than predicted by simple electrostatic interaction models. A more complex model, based on the concept of random potential fluctuations modifying then the energy spectrum of impurities [7, 17], does not fit the trend of our data.

\section{Mobility analysis}

Considering the degenerate band structure of heavy- and light-holes, only an approximate analysis of the mobility for $p$-type III-V compounds is feasible. An extensive review of the approaches needed to calculate the mobility analytically in $p$-type III-V compounds has been given by Wiley [18]. Two models of different sophistication were used in the analysis.

In the first and simpler heuristic model, following Wiersma et al. [19] it was assumed that the temperature dependence of the hole mobility can be described using a semi-empirical approach

$$
1 / \mu=\sum_{(i)} 1 / \mu_{0 i}\left(T / T_{0}\right)^{n_{\mathrm{i}}},
$$

where the characteristic exponents $n_{\mathrm{i}}$ can take integer or half-integer values. Typical results of such fits are shown in Fig. 4. Besides lattice scattering (with $n_{\mathrm{i}}=-3 / 2$, and $\mu_{\mathrm{L}}(300) \approx 900 \mathrm{~cm}^{2} /(\mathrm{V} \mathrm{s})$, deduced from empirical data from the literature [20-22]) and ionized impurity scattering $\left(n_{\mathrm{i}}=3 / 2\right)$, the inclusion of an additional scattering mechanism characterized with $n_{\mathrm{i}}=-1 / 2$ (ascribed to scattering on space charge regions [23]) was found necessary for the fits. The strength of the ionized impurity scattering necessary to account for the low temperature 


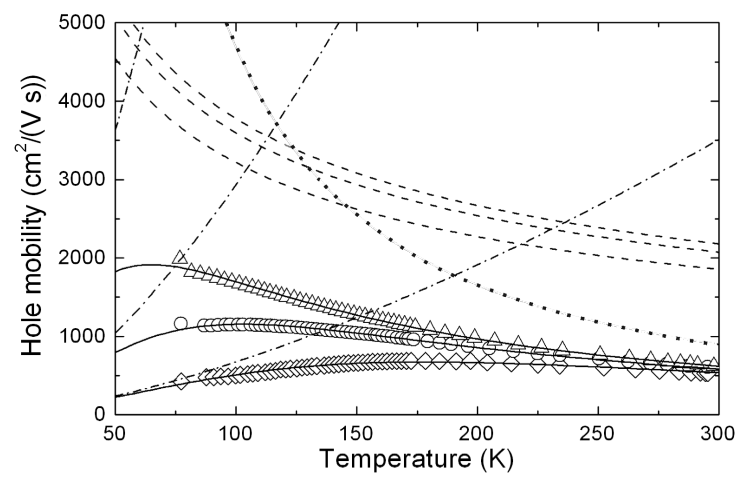

Fig. 4. Fit of the heuristic mobility model to the experimental data. Dot line lattice $\left(\sim T^{-3 / 2}\right)$, dash lines — space charge $\left(\sim T^{-1 / 2}\right)$, dash-dot lines — ionized impurity $\left(\sim T^{3 / 2}\right)$, full lines — total mobility.

mobility was much less than implied by the acceptor and donor concentrations deduced from the Hall fits.

In the second case a phenomenological two-hole band model (parabolic and spherical heavy- and light-hole bands) conceived by Wiley [18] was used, including overlap corrections and interband transitions. Besides lattice scattering (polar and non-polar optical phonon, deformation potential coupled acoustic phonon, and piezoelectric scattering), ionized impurity scattering in the Conwell-Weisskopf formulation and space charge scattering were considered. The total mobility was calculated using the Matthiesen rule. Relevant expressions for the various mobility contributions in the two-hole band model are found [18] except for piezoelectric and space charge scattering, for which the two-hole band formulae were taken from [24]. For strong compensation, as here, screening of ionized impurities by other impurities implied in the Conwell-Weisskopf approach seems to be more appropriate than the free carrier screening used in the Brooks-Herring method.

Material parameters used in the mobility calculations are collected in Table II. Figure 5 shows that the lattice mobility calculated using theoretically estimated deformation potential constants $E_{\mathrm{AC}}=3.6 \mathrm{eV}$ and $E_{\mathrm{NPO}}=5.9 \mathrm{eV}$ fits well the highest reported data in the temperature range of 200-450 K.

A typical mobility fit result is presented in Fig. 6. The full two-band hole mobility model is also resulted in an acceptable fit only if a partial mobility with $\sim T^{-1 / 2}$ like temperature dependence was included with much lower ionized impurity concentration values than the sum of ionized acceptor and donor concentrations obtained from the Hall fit.

Two possible mechanisms, i.e. space-charge scattering [23] and dipole scattering $[28,29]$ could possibly account for the $\mu \sim T^{-1 / 2}$ mobility term. Both express in a certain respect the limitations of the standard single scattering event ionized impurity scattering model. In both cases the strength of the ionized impurity scattering necessary to account for the low temperature mobility was much 
TABLE II

Material parameters for GaSb used in the mobility calculations.

\begin{tabular}{l|c|c|c}
\hline \hline Parameter & Units & Value & Reference \\
\hline Heavy hole effective mass, $m_{\mathrm{hh}} / m_{0}$ & & 0.49 & 18,25 \\
Light hole effective mass, $m_{\mathrm{lh}} / m_{0}$ & & 0.046 & 18,25 \\
Low frequeny dielectric constant, $\varepsilon_{\mathrm{st}}$ & & 15.69 & 18 \\
High frequeny dielectric constant, $\varepsilon_{\mathrm{opt}}$ & & 14.44 & 18 \\
Longitudinal optical phonon temperature, $\Theta_{\mathrm{D}}$ & $\mathrm{K}$ & 347 & 18 \\
Optical deformation potential, $E_{\mathrm{NPO}}$ & $\mathrm{eV}$ & 5.9 & 18 \\
Longitudinal elastic constant, $c_{\mathrm{l}}$ & $\mathrm{N} \mathrm{m}^{-2}$ & $10.367 \times 10^{10}$ & 18,26 \\
Transveres elastic constant, $c_{\mathrm{t}}$ & $\mathrm{N} \mathrm{m}^{-2}$ & $3.5554 \times 10^{10}$ & 18,26 \\
Acoustic deformation potential, $E_{\mathrm{AC}}$ & $\mathrm{eV}^{-2}$ & 3.6 & 18 \\
Piezoelectric constant, $e_{14}$ & $\mathrm{~A} \mathrm{~s} \mathrm{~m}$ & 0.126 & 27
\end{tabular}

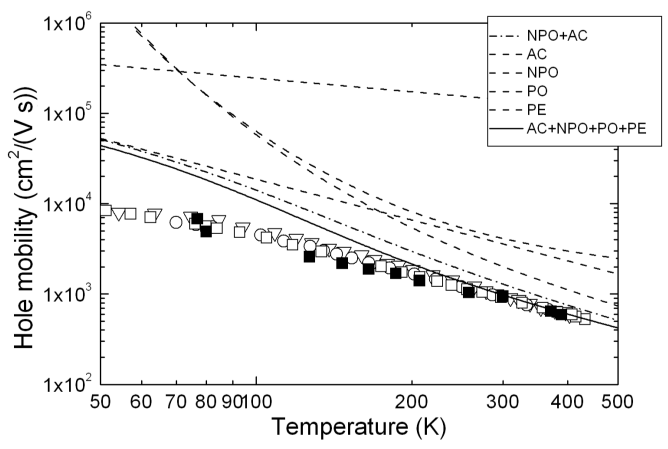

Fig. 5. Comparison of the theoretical two-hole band lattice mobility with experimental data from the literature. Theoretical mobility: AC - acoustic phonon scattering, NPO - nonpolar optical phonon scattering, AC+NPO - combined acoustic and nonpolar optical phonon scattering, PO — polar optical phonon scattering, $\mathrm{PE}$ - piezoelectric scattering, and $\mathrm{AC}+\mathrm{NPO}+\mathrm{PO}+\mathrm{PE}$ - all four lattice scattering mechanisms combined. Experimental data: full squares - [14], open squares - [20], open circles — [21], open triangles - [22].

less than implied by the acceptor and donor concentrations deduced from the Hall fits. Because of the high compensation degree $(K \geq 0.5)$ the effectiveness of the Coulomb scattering by single impurities is much reduced due to correlation between the positions of the scattering centres, and is converted to much less effective space charge region and dipole scattering, both described by a similar temperature dependence.

Considering only scattering on space charge regions, and using the simplest model, the fitted values of the product of $N_{\mathrm{SC}}$, the density of impenetrable spheres and of $Q$, their scattering cross section are $Q N_{\mathrm{SC}}=(1-1.5) \times 10^{5} \mathrm{~cm}^{-1}$ 


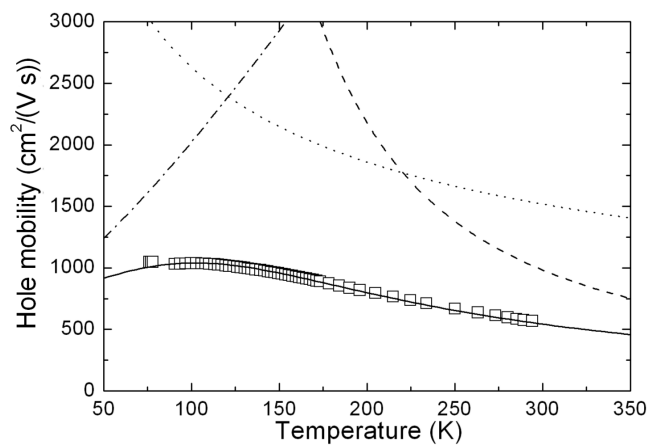

Fig. 6. Two-hole band fit to experimental data. Dot line - lattice scattering, dash-dot line - ionized impurity scattering, dash line - space charge scattering, full line - combined theoretical mobility.

(c.f. Table I). Reliable numerical estimations for the case of dipole scattering are more difficult to make, because their scattering strength depends on the square of the mutual distance of the oppositely charged ions in the dipole.

\section{Conclusions}

The observed differences in the thermal and optical ionization energies of the native acceptors in GaSb in a function of the impurity concentration and compensation were satisfactorily described using a simple Coulomb interaction model. However, the empirically determined constant of proportionality is a factor of 1.4-1.7 larger than the theoretical values. The experimentally determined thermal ionization energies exhibit a somewhat stronger decrease with increasing impurity concentration and compensation degree than predicted in the theory.

The temperature dependence of the hole mobility was analyzed using two models, a simpler heuristic one, and a more sophisticated two-hole band model. Both models gave essentially the same results. The temperature dependence of the hole mobility could be interpreted by considering the dominance space charge and/or dipole scattering over ionized impurity scattering in the case of strong compensation.

\section{Acknowledgments}

The contribution of Dr. K. Somogyi to this work is gratefully acknowledged. This research was supported in part by a grant from the (Hungarian) National Research Fund (OTKA) project No. T 048696.

\section{References}

[1] P.S. Dutta, H.L. Bhat, V. Kumar, J. Appl. Phys. (Appl. Phys. Rev.) 81, 5821 (1997).

[2] W. Jakowetz, W. Rühle, K. Breuninger, Phys. Status Solidi A 12, 169 (1972). 
[3] Landolt-Börnstein, Series III, Vol. 17c, Semiconductors, Eds. O. Madelung, M. Schulz, H. Weiss, Springer, Berlin 1984.

[4] M. Hársy, T. Görög, E. Lendvay, F. Koltay, J. Cryst. Growth 53, 234 (1981).

[5] I. Gyúró, B. Pődör, V.V. Popov, R.V. Parfenev, Phys. Status Solidi A 105, K129 (1988).

[6] J.S. Blakemore, Semiconductor Statistics, Pergamon Press, Oxford 1962.

[7] B.I. Shklovskii, A.L. Efros, Electronic Properties of Doped Semiconductors, Springer-Verlag, Berlin 1984.

[8] A.G. Zabrodskii, M.P. Timofeev, Fiz. Tekh. Poluprovodn. 21, 2217 (1987).

[9] B. Pődör, Phys. Status Solidi A 119, K135 (1990).

[10] G.L. Pearson, J. Bardeen, Phys. Rev. 75, 865 (1949).

[11] P.P. Debye, E.M. Conwell, Phys. Rev. 93, 693 (1954).

[12] J. Monecke, W. Siegel, E. Ziegler, G. Kuhnel, Phys. Status Solidi B 103, 269 (1981).

[13] C.D. Kourkoutas, G.J. Papaioannou, P.C. Euthymiou, G.E. Zardas, Solid State Commun. 67, 651 (1988).

[14] A.N. Baranov, T.I. Voronina, T.S. Lagunova, I.N. Timchenko, Z.I. Chugueva, V.V. Sherstnev, Y.P. Yakovlev, Fiz. Tekh. Poluprovodn. 23, 780 (1989).

[15] P. Doubrava, E. Kucys, F. Moravec, Crystal Preparation and Properties 19-20, 59 (1989).

[16] M.K. Lui, C.C. Ling. X.D. Chen, K.W. Cheah, K.F. Li, in: Materials Research Society Symposium Proc., Vol. 799, Materials Research Society, Boston 2004, Z5.29.1.

[17] N.V. Lien, B.I. Shklovskii, Fiz. Tekh. Poluprovodn. 13, 1763 (1979).

[18] J.D. Wiley, in: Semicondcutors and Semimetals, Eds. R.K. Willardson, A.C. Beer, Vol. 10, Academic Press, New York 1975, p. 91.

[19] R.D. Wiersma, J.A. Stotz, O.J. Pitts, C.X. Wang, M.L. W. Thewalt, S.P. Wattkins, Phys. Rev. B 67, 165202 (2003).

[20] F.J. Reid, R.D. Baxter, S.E. Miller, J. Electrochem. Soc. 113, 713 (1966).

[21] G.R. Johnson, B.C. Cavenett, T.M. Kerr, P.B. Kirby, C.E.C. Wood, Semicond. Sci. Technol. 3, 1167 (1988).

[22] A. Baraldi, C. Ghezzi, R. Magnanini, A. Parisini, L. Tarricone, A. Bosacchi, S. Franchi, V. Avanzini, Mater. Sci. Eng. B 28, 174 (1994).

[23] R.L. Weisberg, J. Appl. Phys. 33, 1817 (1962).

[24] S. Adachi, Physical Properties of III-V Semiconductor Compounds, Wiley, New York 1992.

[25] P. Lawaetz, Phys. Rev. B 4, 3460 (1971).

[26] W.F. Boyle, R.J. Sladek, Phys. Rev. B 11, 2933 (1975).

[27] G. Arlt, P. Quadflieg, Phys. Status Solidi 25, 323 (1968).

[28] R. Stratton, J. Phys. Chem. Sol. 23, 1011 (1962).

[29] B.K. Ridley, Quantum Processes in Semiconductors, 2nd ed., Clarendon Press, Oxford 1988. 